\title{
Absence of Bound States in Extremely Asymmetric Positive Diatomic Molecules
}

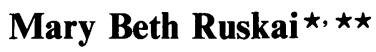 \\ Department of Mathematics, University of Lowell, Lowell, MA 01854, USA
}

\begin{abstract}
It is shown that the Hamiltonian for a diatomic molecule consisting of $N$ electrons and two dynamic nuclei with charges $Z_{1}$ and $Z_{2}$ has no bound states if one of the charges is sufficiently large. The nuclear motion is completely unrestricted, and the kinetic energy of both nuclei can be included in the Hamiltonian. One of the nuclear charges can be arbitrarily small, provided that the other is sufficiently large.
\end{abstract}

\section{Introduction}

For atomic systems, it is well-known that all positive ions are so stable that they have infinitely many bound states [1-4], but that extremely negative ions are unstable with respect to expulsion of at least one electron [5-8]. (See [9] for a complete, up-to-date list of references.) However, one expects that molecular systems will be unstable if the nuclear charges are either very large, or very small, relative to the number of electrons. Proofs of both of these phenomena were recently given $[9,10]$ for diatomic molecules in which the nuclei are completely dynamic, i.e., the nuclear motion is unrestricted and the kinetic energy of the nuclei is included in the Hamiltonian. In particular, it was shown in [10] that a positive diatomic molecule will be unstable with respect to breakup into atomic subsystems when both nuclear charges are large compared to the number of electrons; however, this argument was unable to preclude the existence of bound states for systems in which one of the nuclear charges was less than the total number of electrons, even if the other charge was absurdly large. In this paper we remedy this defect, i.e., it is shown that a positive diatomic molecule is unstable if one of the nuclear charges is sufficiently large.

$\star$ Research supported in part by NSF grant DMS-8908125

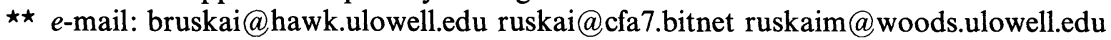


Considerably sharper results can be obtained if approximations which restrict the nuclear dynamics are used. Solovej [11] studied diatomic molecules in the Born-Oppenheimer approximation, and proved that they are asymptotically neutral; this result can be extended [12] to polyatomic systems in which the number of nuclei and their charge ratios are fixed. Chen and Spruch [13] studied small molecules in the adiabatic approximation. For one-electron systems, with nuclear charges $Z_{1} \geqq 1$ and $Z_{2} \geqq 1$, they showed absence of bounded states if $Z_{1}+Z_{2} \geqq 3$; for homonuclear two-electron systems, they showed absence of bound states if $Z_{1}=Z_{2} \geqq 3$. However, their techniques do not seem to provide any information when one of the nuclear charges is smaller than the number of electrons.

By contrast, the results of this paper hold even if one of the charges is extremely small. Let $H^{\mathrm{mol}}$ be the Hamiltonian for a diatomic molecule with $N$ electrons, nuclear charges $Z_{1}$ and $Z_{2}$, and nuclear masses $M_{1}$ and $M_{2}$ satisfying $M_{k}=Z_{k} m_{k}$, where $m_{1}$ and $m_{2}$ are constants. Our main result can be stated as follows.

Theorem. For every fixed pair of values $Z_{2}$ and $N$, there exists a constant $Z_{1}^{c}>0$ such that whenever $Z_{1}>Z_{1}^{c}$, then $H^{\mathrm{mol}}$ has no discrete spectrum.

The dependence of $Z_{1}^{c}$ on $N, Z_{2}$, and $M_{k}$ is discussed in Sect. III. The results are not only far from optimal, they are, at least for very small $Z_{2}$, dominated by the need to control the localization error. Thus, the estimates obtained here seem to be an artifact of the techniques used in the proof and need not have any physical significance. It should be noted that the nuclear masses do not have to be large, so that one or both of the positive particles in the "molecule" could be a positron or meson-like particle; and the assumption $M_{k}=Z_{k} m_{k}$ can be weakened. The only essential requirement is that the mass of the larger nucleus grow at least as fast as its charge $Z_{1}$. The existence of $Z_{1}^{c}$ does not require any conditions on $M_{2}$ except that it be non-zero; however, as discussed in Sect. III, the estimates on $Z_{1}^{c}$ do depend on $M_{2}$. Although the analysis presented here was motivated by the desire to treat the case $Z_{2}<N$, it is equally valid for $Z_{2}>N$. Moreover, the estimates on $Z_{1}^{c}$ in the latter case are better than those in $[10]$ when the charge ratio $Z_{1} / Z_{2}$ is large, i.e., $Z_{1} / Z_{2}>O\left(N^{1 / 3}\right)$.

The proof uses a variant of the localization techniques (discussed under the sobriquet "geometric methods" in Cycon et al. [8]) which are often useful in bound state problems. The particular localization used here was introduced earlier $[9,10]$ for molecular systems. The new ingredient of this paper is not the localization, but the inclusion of estimates on differences in threshold energies.

Both the notation and localization closely follow that used in [9] and differ slightly from that in [10]. Let $\mathbf{R}_{1}$ and $\mathbf{R}_{2}$ denote the positions of the two nuclei, $\xi_{j}$ $(j=1 \ldots N)$ the coordinates of the electrons, $m$ and $M_{k}(k=1,2)$ the electron and nuclear masses, respectively, and $Z_{k}(k=1,2)$ the nuclear charges. After removal of the center of mass motion, we choose as coordinates $\mathbf{R}=\mathbf{R}_{12}=\mathbf{R}_{1}-\mathbf{R}_{2}$ and $x_{i}$ $(i=1 \ldots N)$, where $\mathbf{x}_{i}=\xi_{i}-\mathbf{R}_{N}$ is the position of the $k^{\text {th }}$ electron relative to the nuclear center of mass $\mathbf{R}_{N}=\mu_{1} \mathbf{R}_{1}+\mu_{2} \mathbf{R}_{2}$ and $\mu_{k}=\frac{M_{k}}{M_{1}+M_{2}}$. It will be convenient to denote the internuclear distance by $R=\left|\mathbf{R}_{12}\right|$, and the nucleus-electron distances by $\xi_{j 1} \equiv\left|\xi_{j}-\mathbf{R}_{1}\right|=\left|\mathbf{x}_{j}-\mu_{2} \mathbf{R}_{12}\right|$, and $\xi_{j 2} \equiv\left|\xi_{j}-\mathbf{R}_{2}\right|=\left|\mathbf{x}_{j}+\mu_{1} \mathbf{R}_{12}\right|$. In this notation, the Hamiltonian of a diatomic molecule with the center of mass motion removed 
can be written as

$$
\begin{aligned}
H^{\mathrm{mol}}\left(N, M_{k}, Z_{k}\right)= & -\frac{M_{1}+M_{2}}{2 M_{1} M_{2}} \Delta_{\mathbf{R}}-\frac{1}{2 m} \sum_{i=1}^{N} \Delta_{i}-\frac{1}{2\left(M_{1}+M_{2}\right)}\left|\sum_{i=1}^{N} \nabla_{i}\right|^{2} \\
& -\sum_{i=1}^{N}\left(\frac{Z_{1}}{\xi_{i 1}}+\frac{Z_{2}}{\xi_{i 2}}\right)+\sum_{i<j} \frac{1}{\left|\mathbf{x}_{i}-\mathbf{x}_{j}\right|}+\frac{Z_{1} Z_{2}}{R}
\end{aligned}
$$

where $\Delta_{i}$ denotes $\Delta_{\mathbf{x}_{i}}$, and $\left|\sum_{i=1}^{N} \nabla_{i}\right|^{2}$ is referred to as the Hughes-Eckart term. Recall that $H^{\mathrm{mol}}$ acts on a suitable domain $\mathscr{D}$ of smooth, square-integrable functions which are antisymmetric in the electron coordinates, $\left(\mathbf{x}_{i}, s_{i}\right)$, where $s_{i}$ denotes spin (which will be suppressed since it does not play an essential role). In all that follows, $Z_{1}$ always denotes the larger charge.

Let $\alpha=\left(\alpha_{1}, \alpha_{2}\right)$ be a partition of $\{1 \ldots N\}$ into two disjoint sets (one of which may be empty). The total system can be partitioned into two clusters corresponding to the first nucleus together with those electrons for which $i \in \alpha_{1}$ and the second nucleus together with those electrons for which $i \in \alpha_{2}$. The cluster Hamiltonian $H_{\alpha}$ is given by

$$
H_{\alpha}=H^{\mathrm{mol}}-I_{\alpha}-M_{\alpha} \Delta_{\alpha}=H^{\mathrm{at}}\left(N_{1}, Z_{1}, M_{1}\right)+H^{\mathrm{at}}\left(N_{2}, Z_{2}, M_{2}\right),
$$

where $N_{k}$ denotes the number of elements in $\alpha_{k}, H^{\text {at }}\left(N_{k}, Z_{k}, M_{k}\right)$ is the Hamiltonian of an atom with $N_{k}$ electrons and a nucleus of charge $Z_{k}$ and mass $M_{k}, M_{\alpha} \Delta_{\alpha}$ describes the relative motion of the two atomic clusters, and $I_{\alpha}$ is the intercluster potential

$$
I_{\alpha}=-\sum_{i \in \alpha_{1}} \frac{Z_{2}}{\xi_{i 2}}-\sum_{j \in \alpha_{2}} \frac{Z_{1}}{\xi_{j 1}}+\frac{Z_{1} Z_{2}}{R}+\sum_{i \in \alpha_{1}} \sum_{j \in \alpha_{2}} \frac{1}{\left|\mathbf{x}_{i}-\mathbf{x}_{j}\right|} .
$$

In this notation, it follows from the well-known $\operatorname{HVZ}$ theorem $[3,4,8]$ that

$$
\begin{aligned}
\inf & \left\{\sigma_{\mathrm{ess}}\left(H^{\mathrm{mol}}\right)\right\} \equiv E_{*}\left(H^{\mathrm{mol}}\right) \leqq E^{*} \equiv \min _{\alpha}\left[\inf \left\{\sigma\left(H_{\alpha}\right)\right\}\right] \\
& =\min _{\alpha}\left\{E_{0}\left(H_{\alpha}\right)\right\}=\min _{\alpha}\left\{E_{0}\left[H^{\mathrm{at}}\left(N_{1}, Z_{1}, M_{1}\right)\right]+E_{0}\left[H^{\mathrm{at}}\left(N_{2}, Z_{2}, M_{2}\right)\right]\right\},
\end{aligned}
$$

where $\sigma$ and $\sigma_{\text {ess }}$ denote the spectrum and essential spectrum of the indicated Hamiltonians, $E_{0}(H)$ and $E_{*}(H)$ denote their respective infimums, and $E^{*}$ is defined in (4). It is elementary [see (A-1) to (A-4) and (15)] to show that if $Z_{1}$ is sufficiently large, the minimum above will occur when all electrons are associated with the larger nucleus, i.e., $N_{1}=N$, so that

$$
E^{*}=E_{0}\left[H^{\text {at }}\left(N, Z_{1}, M_{1}\right)\right] \text {. }
$$

In fact, we will show that if $Z_{1}$ is sufficiently large, then

$$
\left\langle\Psi, H^{\mathrm{mol}} \Psi\right\rangle \geqq E_{0}\left[H^{\text {at }}\left(N, Z_{1}, M_{1}\right)\right]\|\Psi\|^{2} \quad \forall \Psi \text { in } \mathscr{D}\left(H^{\mathrm{mol}}\right)
$$

so that equality holds in (4) and

$$
E_{0}\left(H^{\mathrm{mol}}\right)=E_{*}\left(H^{\mathrm{mol}}\right)=E^{*}=E_{0}\left[H^{\mathrm{at}}\left(N, Z_{1}, M_{1}\right)\right] \text {. }
$$

This paper has three appendices. A number of elementary, but important, results about atomic systems are summarized in Appendix A. In order to make this paper more accessible, if not completely independent of $[9,10]$, the construction of the localizing functions is sketched in Appendix B. Finally, in Appendix C, the 
proof is described for the special, and somewhat simpler, case of diatomic molecules with only one electron. Those who wish to grasp the underlying ideas with a minimum of technical detail may prefer to read this appendix first.

\section{Proof}

To prove that $H^{\mathrm{mol}}$ has no discrete spectrum, it suffices to find a partition of unity $\left\{F_{\alpha}\right\}$ such that, if $Z_{1}$ is sufficiently large, then $\forall \Psi \in \mathscr{D}\left(H^{\mathrm{mol}}\right)$

$$
\left\langle\Psi, H^{\mathrm{mol}} \Psi\right\rangle=\sum_{\alpha}\left\langle F_{\alpha} \Psi,\left[H^{\mathrm{mol}}-L E\right] F_{\alpha} \Psi\right\rangle \geqq E^{*}\|\Psi\|^{2},
$$

where the localization error $L E$ is given by the well-known formula

$$
L E=\sum_{\alpha} F_{\alpha} H^{\mathrm{mol}} F_{\alpha}-H^{\mathrm{mol}}=\sum_{\mu} \sum_{\alpha} c_{\mu}\left|\nabla_{\mu} F_{\alpha}\right|^{2},
$$

where $\sum_{\mu}$ indicates a sum over all gradients for which a corresponding Laplacian (including the Hughes-Eckart term) appears $H^{\mathrm{mol}}$ and $c_{\mu}$ denotes its coefficient.

It was shown previously $[9,10]$ that for any choice of $\varepsilon>0$ and $\varrho>0$ one can find a set of localizing functions $\left\{F_{\alpha}\right\}$, indexed by cluster decompositions $\alpha$, and an additional function $F_{0}$, with the following properties:

a) $\sum_{\alpha=0}^{2^{N}} F_{\alpha}^{2}=1$

b) $F_{0}$ is symmetric with respect to interchange of all electrons, and

$$
\operatorname{supp} F_{0} \equiv\left\{(\mathbf{x}, \mathbf{R}): F_{0}(\mathbf{x}, \mathbf{R}) \neq 0\right\} \subset\{(\mathbf{x}, \mathbf{R}): R<(1+\varepsilon) \varrho\} \text {. }
$$

c) $F_{\alpha}$ is symmetric with respect to interchange of coordinates of electrons within $\alpha_{1}$ or $\alpha_{2}$, and

d)

$$
\begin{aligned}
\operatorname{supp} F_{\alpha} \equiv & \left\{(\mathbf{x}, \mathbf{R}): F_{\alpha}(\mathbf{x}, \mathbf{R}) \neq 0\right\} \\
& \subset\left\{(\mathbf{x}, \mathbf{R}): \xi_{i 1}<(1+\varepsilon) \xi_{i 2} \forall i \in \alpha_{1}, \xi_{i 2}<(1+\varepsilon) \xi_{i 1} \forall i \in \alpha_{2}, \text { and }(1+\varepsilon) R>\varrho\right\} .
\end{aligned}
$$

e) The localization error satisfies

$$
\begin{aligned}
L E & \leqq \frac{A_{0}}{\varepsilon^{2} M_{2}} \frac{1}{\varrho^{2}} F_{0}^{2}+\frac{1}{\varepsilon^{2}} \frac{1}{\varrho R} \sum_{\alpha \neq 0}\left[\frac{B_{1} N^{2}}{M_{2}}+\frac{B_{2} N^{2}}{M_{1}+M_{2}}+\frac{B_{3}}{m}\right] F_{\alpha}^{2} \\
& \leqq \frac{A_{0}}{\varepsilon^{2} M_{2}} \frac{1}{\varrho^{2}} F_{0}^{2}+\frac{A N^{2}}{\varepsilon^{2} M_{2}} \frac{1}{\varrho R} \sum_{\alpha \neq 0} F_{\alpha}^{2} \equiv \sum_{\alpha} L_{\alpha} F_{\alpha}^{2},
\end{aligned}
$$

where $A_{0}, A$, and $B_{k}$ are constants, (10) defines $L_{\alpha}$ and we have used the fact that (for sufficiently large $\left.Z_{1}\right) M_{2} \leqq M_{1}$ so that $\frac{1}{M_{2}} \geqq \frac{M_{1}+M_{2}}{2 M_{1} M_{2}}$.

Property d), which is critical to our analysis, says that, roughly speaking, electrons in the cluster $\alpha_{1}$ are closer to the first nucleus and those in $\alpha_{2}$ to the second. It further follows from the triangle inequality that electrons in a cluster are bounded away from the opposite nucleus by a distance of about $\frac{R}{2}$, i.e.,

$$
\operatorname{supp} F_{\alpha} \subset\left\{(\mathbf{x}, \mathbf{R}): R<(2+\varepsilon) \xi_{i 2} \forall i \in \alpha_{1} \text {, and } R<(2+\varepsilon) \xi_{i 1} \forall i \in \alpha_{2}\right\}
$$


To return to the proof, it is useful to observe that (8) is equivalent to

$$
\left\langle F_{\alpha} \Psi,\left[H^{\mathrm{mol}}-L_{\alpha}-E^{*}\right] F_{\alpha} \Psi\right\rangle \geqq 0 \quad \forall \alpha .
$$

The cases $\alpha=0$ and $\alpha \neq 0$ are treated differently.

For the $\alpha=0$ case, the united atom bound (A-9) is useful. It then follows from property b) above and (10) that

$$
\begin{aligned}
& \left\langle F_{0} \Psi,\left[H^{\mathrm{mol}}-L_{0}-E^{*}\right] F_{0} \Psi\right\rangle \\
& \quad \geqq\left\langle F_{0} \Psi,\left[-\delta Z_{1} Z_{2} N^{1 / 3}+\frac{Z_{1} Z_{2}}{(1+\varepsilon) \varrho}-\frac{A_{0}}{\varepsilon^{2} M_{2}} \frac{1}{\varrho^{2}}\right] F_{0} \Psi\right\rangle,
\end{aligned}
$$

where we have used (A-8) and the fact that

$$
E_{0}\left[H^{U A}\right]-E^{*}=E_{0}\left[H^{\mathrm{at}}\left(N, Z_{1}+Z_{2}, M_{1}+M_{2}\right)\right]-E_{0}\left[H^{\mathrm{at}}\left(N, Z_{1}, M_{1}\right)\right] .
$$

If $Z_{1} Z_{2}>4 \delta N^{1 / 3}\left(\frac{1+\varepsilon}{\varepsilon}\right)^{2} \frac{A_{0}}{M_{2}}$, one can find a positive constant $d$ such that the right side of (13) will be positive if $\frac{1}{\varrho}=d N^{1 / 3}$. Thus, if $Z_{1}$ is sufficiently large, (12) holds for $\alpha=0$. However, this requires $Z_{1}>\frac{c}{Z_{2} M_{2}}=O\left(Z_{2}^{-2}\right)$ [with $N$ fixed and $N^{1 / 3}$ absorbed into the constant $c$ ] so that if $Z_{2}$ is extremely small, $Z_{1}$ must be huge.

Verification of (12) for $\alpha \neq 0$ constitutes the heart of the proof. First, observe that $H^{\mathrm{mol}}>H_{\alpha}+I_{\alpha}$ and use (11) to bound the intercluster potential $I_{\alpha}$ to conclude that

$$
\begin{aligned}
&\left\langle F_{\alpha} \Psi,\left[H^{\mathrm{mol}}-L_{\alpha}-E^{*}\right] F_{\alpha} \Psi\right\rangle \\
& \geqq\left\langle F_{\alpha} \Psi,\left[E_{0}\left(H_{\alpha}\right)-E^{*}-\frac{(2+\varepsilon) N_{2} Z_{1}}{R}-\frac{(2+\varepsilon) N_{1} Z_{2}}{R}\right.\right. \\
&\left.\left.+\frac{Z_{1} Z_{2}}{R}-\frac{A N^{2}}{\varepsilon^{2} M_{2}} \frac{1}{\varrho R}\right] F_{\alpha} \Psi\right\rangle .
\end{aligned}
$$

Previously (in $[9,10])$, the positive difference in threshold energies $E_{0}\left(H_{\alpha}\right)-E^{*}$ was discarded; however, in order to treat asymmetric molecules with $Z_{2}<N<Z_{1}$, it is essential that this quantity be retained. It can be bounded using (A-4) as

$$
\begin{aligned}
& E_{0}\left(H_{\alpha}\right)-E^{*} \\
& \quad=E_{0}\left[H^{\mathrm{at}}\left(N_{1}, Z_{1}, M_{1}\right)\right]-E_{0}\left[H^{\mathrm{at}}\left(N, Z_{1}, M_{1}\right)\right]+E_{0}\left[H^{\mathrm{at}}\left(N_{2}, Z_{2}, M_{2}\right)\right] \\
& \quad \geqq \beta \frac{N_{2} Z_{1}^{2}}{N^{2 / 3}}-\sigma Z_{2}^{2} N_{2}^{1 / 3} .
\end{aligned}
$$

Although this certainly suggests that (14) will be positive if $Z_{1}$ is sufficiently large, a complete proof requires a comparison between this expression and the terms involving $\frac{1}{R}$ in (14). As Solovej [11] observed, the ground state wave function $\Psi$ satisfies (A-10), i.e.,

$$
\left\langle\Psi, \frac{1}{R} \Psi\right\rangle \leqq \delta N^{1 / 3}\|\Psi\|^{2} .
$$

However, we were unable to establish that this inequality still holds if $\Psi$ is replaced by $F_{\alpha} \Psi$. Therefore, we will separately consider two situations. 
First, suppose that the somewhat weaker (for large $Z_{1}$ ) inequality

$$
\left\langle F_{\alpha} \Psi, \frac{1}{R} F_{\alpha} \Psi\right\rangle \leqq Z_{1}^{1 / 3}\left\|F_{\alpha} \Psi\right\|^{2}
$$

holds. Then, if the small term in (15) involving $Z_{2}^{2}$ is neglected,

$$
\begin{aligned}
& \left\langle F_{\alpha} \Psi,\left[H^{\mathrm{mol}}-L_{\alpha}-E^{*}\right] F_{\alpha} \Psi\right\rangle \\
& \geqq\left\langle F_{\alpha} \Psi, \frac{1}{R}\left[\beta \frac{N_{2} Z_{1}^{5 / 3}}{N^{2 / 3}}-(2+\varepsilon) N_{2} Z_{1}-(2+\varepsilon) N_{1} Z_{2}\right.\right. \\
& \left.\quad+Z_{1} Z_{2}-\frac{a N^{7 / 3}}{\varepsilon^{2} M_{2}}\right] F_{\alpha} \Psi,
\end{aligned}
$$

where $a=d A$. Clearly, (17) will be positive if $Z_{1}$ is sufficiently large. To estimate how large, rewrite the terms within the square brackets in the form

$$
N_{2} Z_{1}\left[\beta\left(\frac{Z_{1}}{N}\right)^{2 / 3}-(2+\varepsilon)\right]+Z_{2}\left[Z_{1}-(2+\varepsilon) N_{1}\right]-\frac{a N^{7 / 3}}{\varepsilon^{2} M_{2}} .
$$

The first two sets of terms will be positive if $Z_{1}>c N$, where the constant $c$ is independent of $Z_{2}$; however, control of the localization error requires a much stronger condition on $Z_{1}$ as $N$ increases and/or $Z_{2}$ decreases.

To complete the proof, one must also consider the possibility that inequality (16) fails, i.e.,

$$
\left\langle F_{\alpha} \Psi, \frac{1}{R} F_{\alpha} \Psi\right\rangle \geqq Z_{1}^{1 / 3}\left\|F_{\alpha} \Psi\right\|^{2} .
$$

In this case, the argument above fails, but one can use the united atom bound to conclude, as in (13), that

$$
\begin{aligned}
& \left\langle F_{\alpha} \Psi,\left[H^{\mathrm{mol}}-L_{\alpha}-E^{*}\right] F_{\alpha} \Psi\right\rangle \\
& \geqq\left\langle F_{\alpha} \Psi,\left[-\delta Z_{1} Z_{2} N^{1 / 3}+\frac{Z_{1} Z_{2}}{R}-\frac{A N^{2}}{\varepsilon^{2} M_{2}} \frac{1}{\varrho R}\right] F_{\alpha} \Psi\right\rangle . \\
& \geqq\left\langle F_{\alpha} \Psi,\left[-\delta Z_{1} Z_{2} N^{1 / 3}+Z_{1}^{4 / 3} Z_{2}-\frac{a N^{7 / 3}}{\varepsilon^{2} M_{2}}\right] F_{\alpha} \Psi\right\rangle,
\end{aligned}
$$

where the second inequality follows from (18). Again, this will be positive if $Z_{1}$ is sufficiently large; as before, a linear bound of $Z_{1}>\delta^{3} N$ will suffice if the localization error is ignored. (However, not only must $Z_{1}$ be large enough to control the localization error in the final expression, but one must already have $Z_{1} Z_{2}-\frac{a N^{7 / 3}}{\varepsilon^{2} M_{2}}>0$ to even justify to applying (18)). Thus, irrespective of whether (16) or (18) holds, (12) is true for all $\alpha \neq 0$ if $Z_{1}$ is sufficiently large. This completes the proof.

\section{Discussion}

In order to discuss the dependence of $Z_{1}^{c}$ on such parameters as $N, Z_{2}$ and the nuclear masses, it is useful to summarize all of the conditions that $Z_{1}$ needs to 
satisfy to insure that (8) holds so that $H^{\mathrm{mol}}$ has no discrete spectrum. In all cases, $c$ (or $c^{\prime}$ ) will denote an unspecified positive constant.

a) To insure that (5) holds, i.e., $E_{0}\left[H^{\text {at }}\left(N, Z_{1}, M_{1}\right)\right] \leqq E_{0}\left[H_{\alpha}\right] \forall \alpha$, it suffices that the right side of (15) is positive. This will be true if $Z_{1}>c N^{1 / 3} Z_{2}$. [This condition also suffices to justify neglecting the term involving $Z_{2}^{2}$ in (17).]

b) To insure that it is possible to choose $\varrho$ so that (13) is positive, one needs $Z_{1}>\frac{c N^{1 / 3}}{\varepsilon^{2} M_{2} Z_{2}} \approx \frac{c^{\prime} N^{1 / 3}}{\varepsilon^{2} Z_{2}^{2}}$. However, this is only necessary to control the localization error arising from the nuclear kinetic energy.

c) To insure that (14) is positive, the estimate (A-4) is critical. As discussed in Appendix A, this may require $Z_{1}>N^{2}$.

d) To insure that (17) is positive, one needs only $Z_{1}>c N$, independent of $Z_{2}$, if the localization error is ignored. Otherwise, one needs $Z_{1}>\frac{c N^{7 / 3}}{\varepsilon^{2} M_{2} Z_{2}} \approx \frac{c^{\prime} N^{7 / 3}}{\varepsilon^{2} Z_{2}^{2}}$.

e) To insure that (19) is positive, one needs only $Z_{1}>\delta^{3} N$, if the localization error is ignored. Controlling this requires $Z_{1}>O\left(Z_{2}^{-2}\right)$ as in d).

It is worth making a few remarks about the condition $M_{k}=Z_{k} m_{k}$. For the larger nucleus, it can be replaced by $M_{1} \geqq Z_{1} m_{1}$. This is essential to the validity of (A-8), which is critical to the proof. Without it, one could not even guarantee the positivity of the first two terms in (13) or (19) since $-\delta Z_{1} Z_{2} N^{1 / 3}$ would be replaced by $-\delta Z_{1}^{2} N^{1 / 3}$. However, the assumption $M_{2}=Z_{2} m_{2}$ was only made for convenience and is not necessary to establish the existence of $Z_{1}^{c}$. The validity of (A-8) also requires either $Z_{1} Z_{2}>c M_{2} N$ or $\frac{Z_{2}}{Z_{1}}<\frac{M_{2}}{M_{1}}$, so that absurd growth of $M_{2}$ would alter our estimates. If this is avoided, $Z_{1}=O\left(\left(M_{2} Z_{2}\right)^{-1}\right)$ suffices both to control the localization error, and to insure the validity of the arguments leading to (13) and (19). Thus, if the smaller nucleus has constant mass as $Z_{2} \downarrow 0$, one can conclude that $Z_{1}^{c} \leqq c N Z_{2}^{-1}$ when $Z_{2}<\frac{1}{N}$.

The analysis presented here was primarily concerned with the case $Z_{2}<N$; however, it is also valid when $Z_{2} \geqq N$. Moreover, in the latter case, the terms arising from the localization error become negligible as $N, Z_{2} \rightarrow \infty$ with $M_{k} \geqq Z_{k} m_{k}$ $(k=1,2)$. Therefore, b), d), and e) reduce to $Z_{1}>c N$. Now, as discussed after $(\mathrm{A}-5)$, the condition $Z_{1}>N^{2}$ in c) is actually necessary only if $N_{2}$ is small; otherwise it suffices that $Z_{1}>c N$. However, one could construct a more refined argument (similar to that in [10]) to show that the internuclear repulsion is sufficient to insure that (14) is positive when $N_{2}$ is small compared to both $N$ and $Z_{2}$ (e.g., $5 N_{2} \leqq N \leqq Z_{2}$ ). Thus, c) can be replaced by $Z_{1}>c N$ when $Z_{2} \geqq N$. Finally, condition a) requires $Z_{1}>c N^{4 / 3}$ when $Z_{2} \geqq N$. Since the latter condition is the most restrictive, this yields an asymptotic estimate of $Z_{1}^{c} \leqq c Z_{2} N^{1 / 3}$.

In principle, the results obtained here should be better than those in [10] since the difference in threshold energies was neglected in [10]. Unfortunately, because of the crudity of some of the estimates, this need not be true. In order to make a comparison, it is helpful to rewrite the asymptotic estimate $\frac{1}{N^{c}} \approx \frac{1}{Z_{1}}+\frac{1}{Z_{2}}$ given in [10] as $Z_{1}^{c} \leqq \frac{Z_{2}}{Z_{2}-N} N$. Evidently, the bound given above is better if $Z_{2}$ is close to $N$, i.e., for $N<Z_{2}<N+c^{\prime} N^{2 / 3}<2 N$; however, for larger values of $Z_{2}$ the results of [10] are still superior. To summarize, the results obtained here are sharper when the ratio $Z_{1} / Z_{2}$ is larger; those of [10] are better when $Z_{1} \approx Z_{2}$. 
If the localization error is ignored and $Z_{2}$ is very small, the analysis presented here implies that $Z_{1}^{c} \leqq c N$ independent of $Z_{2}$ even as $Z_{2} \rightarrow 0$. At first, this might seem counter-intuitive. However, when the ratio $Z_{1} / Z_{2}$ is very large, the ground state electron density is concentrated near the larger nucleus, and to a very good approximation the smaller nucleus will see an effective charge of $Z_{1}-N$, which is repulsive for $Z_{1}>N$. Moreover, as $Z_{2} \downarrow 0$ the charge ratio increases dramatically so that polarization effects are minimal and this naive argument eventually becomes accurate. This suggests that $Z_{1}^{c} \approx N+1$ when $Z_{2}$ is below some small critical value. It also suggests that the requirement $Z_{1} \geqq O\left(Z_{2}^{-2}\right)$ is an artifact of the localization error in the proof and of no physical significance. Indeed, the $\frac{1}{M_{2}}$ dependence of the localization error leads to estimates on $Z_{1}^{c}$ which are larger for light nuclei, yet there is no reason to expect a positron to bind if a proton does not. Unfortunately, the affect of the localization error does not arise solely from the kinetic energy of the nuclei. Even if the nuclear kinetic energy is omitted, the localization error from the electron kinetic energy will still give rise to a condition of the form $Z_{1} \geqq O\left(Z_{2}^{-1}\right)$.

Finally, it should be mentioned that the fermionic character of the electrons was used only in estimates involving atomic ground state energies. Therefore, the results can be generalized to bosons provided that terms involving $N^{1 / 3}$ are replaced by $N$. This will only affect the dependence of $Z_{1}^{c}$ on $N$.

\section{Appendix A: Properties of Atomic Ground State Energies}

In this appendix a number of elementary, but useful observations about the ground state energies of atomic systems are collected. Unless otherwise stated, proofs can be found in Chap. 4 of Thirring [4].

First, recall that

$$
E_{0}\left[H^{\mathrm{at}}(N, Z, M)\right]=Z^{2} e\left(N, M, \frac{1}{Z}\right),
$$

where $e(N, M, \gamma)$ is the ground state energy of an atomic Hamiltonian with $N$ electrons, a nucleus with charge $Z=1$ and mass $M$, but with the electron-electron interaction multiplied by $\gamma$. It is well known that $e(N, M, \gamma)$ is monotone increasing and concave in $\gamma$, and that one can find positive constants $\sigma$ and $\hat{\sigma}$ such that

$$
-\hat{\sigma} N^{1 / 3}>e(N, M, \gamma)>e(N, M, 0)>-\sigma N^{1 / 3},
$$

Thirring [4] has given a more precise estimate when $N$ is large. To $O\left(N^{-1 / 3}\right)$

$$
-\left(\frac{3}{2} N\right)^{1 / 3}\left(1-\frac{\gamma N}{2}\right)>e(N, M, \gamma)>-\left(\frac{3}{2} N\right)^{1 / 3} \text {. }
$$

Moreover, one can find a positive constant $\beta$ such that, if $Z$ is sufficiently large,

$$
E_{0}\left[H^{\text {at }}\left(N_{1}, Z, M\right)\right]-E_{0}\left[H^{\text {at }}(N, Z, M)\right]>\beta Z^{2} \frac{N-N_{1}}{N^{2 / 3}} .
$$

To verify this, one could use the fact that $e(N, M, 0)$ satisfies a bound of this form, and $e(N, M, \gamma) \rightarrow e(N, M, 0)$ as $\gamma \rightarrow 0$. In fact, Thirring [4] estimated the upper bound 
in (A-3) by using the ground state of $e(N, M, 0)$ as a trial function for arbitrary $\gamma$ to conclude that, to $O\left(N^{-1 / 3}\right)$,

so that

$$
e(N, M, \gamma)-e(N, M, 0)<\left(\frac{3}{2} N\right)^{1 / 3} \frac{\gamma N}{2}
$$

$$
E_{0}\left[H^{\mathrm{at}}\left(N_{1}, Z, M\right)\right]-E_{0}\left[H^{\mathrm{at}}(N, Z, M)\right]>\left(\frac{3}{2} N\right)^{1 / 3} Z^{2}\left[\frac{N-N_{1}}{N}-\frac{N}{2 Z}\right] .
$$

This yields (A-4) if either $Z>N^{2}$, or $N_{1}<2 N$.

Next, we discuss some important bounds on energy differences which follow from certain concavity properties of atomic energies. In all cases, $\delta$ denotes a suitable positive constant. It was shown in [10] that the concavity and monotonicity of $e(N, M, \gamma)$ in $\gamma$ imply that

$$
E_{0}\left[H^{\mathrm{at}}\left(N, Z_{1}+Z_{2}, M\right)\right]-E_{0}\left[H^{\mathrm{at}}\left(N, Z_{1}, M\right)\right]>-\delta Z_{1} Z_{2} N^{1 / 3} .
$$

To take into account the effect of the nuclear masses, one can similarly use the fact that $E_{0}\left[H^{\text {at }}(N, Z, M)\right]$ is concave and monotone increasing in $\frac{1}{M}$ to show that

$$
\begin{aligned}
& E_{0}\left[H^{\mathrm{at}}\left(N, Z_{1}, M_{1}+M_{2}\right)\right]-E_{0}\left[H^{\mathrm{at}}\left(N, Z_{1}, M_{1}\right)\right] \\
& >\frac{M_{2}}{M_{1}+M_{2}}\left\{E_{0}\left[H^{\mathrm{at}}\left(N, Z_{1}, \infty\right)\right]-E_{0}\left[H^{\mathrm{at}}\left(N, Z_{1}, M_{1}\right)\right]\right\} \\
& \quad>-\delta Z_{1} Z_{2} N^{1 / 3}
\end{aligned}
$$

if $M_{k}=Z_{k} m_{k}$. The right side of this estimate is already comparable to that of (A-6). However, it can be further improved by using the fact that $E_{0}\left[H^{\text {at }}(N, Z, M)\right]$ is actually jointly concave in $\frac{1}{m}$ and $\frac{1}{M}$ from which it follows that [4]

$$
E_{0}\left[H^{\mathrm{at}}(N, Z, \infty)\right]-E_{0}\left[H^{\mathrm{at}}(N, Z, M)\right]>\frac{N m}{M} E_{0}\left[H^{\mathrm{at}}(N, Z, \infty)\right],
$$

so that if $M_{1} \geqq Z_{1} m_{1}$,

$$
E_{0}\left[H^{\mathrm{at}}\left(N, Z_{1}, M_{1}+M_{2}\right)\right]-E_{0}\left[H^{\mathrm{at}}\left(N, Z_{1}, M_{1}\right)\right]>-\delta M_{2} N^{4 / 3} .
$$

Whenever $Z_{1} Z_{2} \geqq M_{2} N$, this can be combined with (A-6) to conclude that

$$
E_{0}\left[H^{\text {at }}\left(N, Z_{1}+Z_{2}, M_{1}+M_{2}\right)\right]-E_{0}\left[H^{\text {at }}\left(N, Z_{1}, M_{1}\right)\right]>-\delta Z_{1} Z_{2} N^{1 / 3} \text {. }
$$

Comparison with a "united atom" described by the Hamiltonian $H^{\mathrm{UA}}=H^{\text {at }}\left(N, Z_{1}+Z_{2}, M_{1}+M_{2}\right)$ is also useful. It is well-known that

$$
H^{\mathrm{mol}}>E_{0}\left[H^{\mathrm{UA}}\right]+\frac{Z_{1} Z_{2}}{R} \quad \forall R .
$$

Solovej [11] observed that this can be combined with (A-8) and

$$
E_{0}\left[H^{\text {at }}\left(N, Z_{1}, M_{1}\right)\right]>E_{0}\left[H^{\mathrm{mol}}\right]
$$

to conclude that the ground state wave-function for $H^{\mathrm{mol}}$ satisfies

$$
\left\langle\Psi, \frac{1}{R} \Psi\right\rangle \leqq \delta N^{1 / 3}\|\Psi\|^{2} .
$$




\section{Appendix B: Construction of the Localizing Functions}

In this appendix, the construction of the partition of unity $\left\{F_{\alpha}\right\}$ used in the proof is sketched. One begins with a smooth, monotone function $g: \mathbf{R}^{+} \rightarrow[0,1]$ satisfying $g^{2}(t)+g^{2}\left(\frac{1}{t}\right)=1$ and $\operatorname{supp} g \subset\left[\frac{1}{1+\varepsilon}, \infty\right]$ for some $\varepsilon>0$. (To verify that such functions exist, note that if $f: \mathbf{R}^{+} \rightarrow[0,1]$, then $g(t)=\frac{1}{\sqrt{2}}\left[f^{2}(t)+1-f^{2}\left(\frac{1}{t}\right)\right]^{1 / 2}$ satisfies the first condition.) Since $g(t)=0$ if $t<\frac{1}{1+\varepsilon}$, it follows that

$$
\begin{gathered}
g\left(\frac{\xi_{i 2}}{\xi_{i 1}}\right) \neq 0 \Rightarrow \xi_{i 1}<(1+\varepsilon) \xi_{i 2} \\
\Rightarrow R<(2+\varepsilon) \xi_{i 2} \text { and }\left|\mathbf{x}_{i}\right|<(1+\varepsilon) \xi_{i 2},
\end{gathered}
$$

where (B-2) follows from (B-1) and the triangle inequality. Similarly,

$$
\begin{gathered}
g\left(\frac{\xi_{i 1}}{\xi_{i 2}}\right) \neq 0 \Rightarrow \xi_{i 2}<(1+\varepsilon) \xi_{i 1} \\
\Rightarrow R<(2+\varepsilon) \xi_{i 1} \text { and }\left|\mathbf{x}_{i}\right|<(1+\varepsilon) \xi_{i 1} .
\end{gathered}
$$

Now define a partition of unity by letting

$$
F_{0}=g\left(\frac{\varrho}{R}\right) \quad \text { and } \quad F_{\alpha}=g\left(\frac{R}{\varrho}\right) \prod_{i \in \alpha_{1}} g\left(\frac{\xi_{i 2}}{\xi_{i 1}}\right) \prod_{j \in \alpha_{2}} g\left(\frac{\xi_{j 1}}{\xi_{j 2}}\right) \quad(\alpha \neq 0) .
$$

It is easy to verify that $\left\{F_{\alpha}\right\}$ satisfies properties a)-c) of Sect. II; property d) follows directly from (B-1) and (B-3), and (11) follows from (B-2) and (B-4).

To verify the bounds on the localization error, first recall that $\frac{\xi_{i 1}}{\xi_{i 2}}=\frac{\left|\mathbf{x}_{i}-\mu_{2} \mathbf{R}\right|}{\left|\mathbf{x}_{i}+\mu_{1} \mathbf{R}\right|}$, where $\mu_{k}=\frac{M_{k}}{M_{1}+M_{2}}$ so that $\mu_{1}+\mu_{2}=1$. It is elementary to verify that

$$
\left.\nabla_{\mathbf{y}}\left(\frac{\left|\mathbf{x} \pm \mu_{2} \mathbf{R}\right|}{\left|\mathbf{x} \mp \mu_{1} \mathbf{R}\right|}\right)\right|^{2}=\frac{|\mathbf{y}|^{2}}{\left|\mathbf{x} \mp \mu_{1} \mathbf{R}\right|^{4}}
$$

for either $\mathbf{y}=\mathbf{x}$ or $\mathbf{y}=\mathbf{R}$. Now, $g(t)=1$ if $t>(1+\varepsilon)$, so that $\sup \left|g^{\prime}(t)\right|=O\left(\varepsilon^{-1}\right)$, and $\operatorname{supp} g^{\prime}(t) \subset\left[\frac{1}{1+\varepsilon},(1+\varepsilon)\right]$. Thus, $\nabla_{\mathbf{y}} g\left(\frac{\xi_{i k}}{\xi_{i k^{\prime}}}\right)=0$ if either $\xi_{i 1} \geqq(1+\varepsilon) \xi_{i 2}$ or $\xi_{i 2}<(1+\varepsilon) \xi_{i 1}$ so that both (B-2) and (B-4) hold whenever this gradient is non-zero. It then follows from (B-6) that

$$
\left|\nabla_{\mathbf{y}} g\left(\frac{\xi_{i k}}{\xi_{i k^{\prime}}}\right)\right|^{2} \leqq C_{\varepsilon}^{2} \frac{|\mathbf{y}|^{2}}{\left|\mathbf{x} \mp \mu_{k} \mathbf{R}\right|^{4}} \leqq C_{\varepsilon}^{2} \frac{b}{R^{2}},
$$

where $b$ and $C_{\varepsilon}$ are constants with $C_{\varepsilon}=O\left(e^{-1}\right)$ and, as above, this holds for both $\mathbf{y}=\mathbf{x}$ and $\mathbf{y}=\mathbf{R}$. It is now straightforward to apply the product rule to $F_{\alpha}$ to establish (10). The only possible sublety arises from counting the number of identical terms, and using the identity $g^{2}\left(\frac{\xi_{i 2}}{\xi_{i 1}}\right)+g^{2}\left(\frac{\xi_{i 1}}{\xi_{i 2}}\right)=1$ to rewrite the result in the form $\sum_{\alpha} L_{\alpha} F_{\alpha}^{2}$. For further discussion of these details see [10]. 


\section{Appendix C: One-Electron Molecules}

The author has frequently been urged to provide a "simple" proof that a oneelectron diatomic molecule cannot be too positive. Although it may not be immediately apparent, the argument presented here actually becomes reasonably simple when specialized to the one-electron case. In fact, when $N=1$ and $Z_{1}=Z_{2}$ an argument similar to that in [10] was already given by Cycon et al. [8] (see Sect. 3.6, "Applications to Atomic Physics: A Warm-Up"); one need only reverse the roles of the nuclei and electrons. In view of this, a separate treatment of the one-electron case in [10] was not warranted. However, the argument in [8] is not amenable to extremely asymmetric nuclei. Moreover, the crucial estimates on differences in threshold energies become exact for one-electron systems. Therefore, it seems worth including a separate discussion of the one-electron case.

The localization error is discussed for completeness; however, the reader seeking insight into the underlying ideas may easily ignore this aspect. The essential point of the localization is that configuration space can be decomposed into three nearly disjoint regions which can be described as follows: a region on which the internuclear distance is small, one on which the electron is closer to the larger nucleus, and a third for which the electron is closer to the smaller nucleus. These conditions then imply simple bounds on the expectations of certain potentials. Although the cluster notation introduced previously is not really needed here, we do refer to it after (C-11) so that the ideas presented here give more insight into the general $N$-electron case. Unless otherwise stated, the notation of the introduction is used.

Let $h(M, Z)=\frac{m+M}{2 m M} \Delta_{\mathbf{x}}-\frac{Z}{|\mathbf{x}|}$ denote the Hamiltonian (with center of mass motion removed) of an electron with mass $m$ in the field of a nucleus with charge $Z$ and mass $M$. Recall that $E_{0}[h(M, Z)]=-\frac{m M}{2(m+M)} Z^{2} \approx-\frac{m}{2} Z^{2}$, when $M$ is large. Since $\left|E_{0}\left[h\left(M_{1}+M_{2}, Z_{1}\right)\right]-E_{0}\left[h\left(M_{1}, Z_{1}\right)\right]\right|<\left(\frac{m}{m_{1}}\right)^{2} M_{2}$ will be negligible compared to $Z_{1} Z_{2}$, we will henceforth suppress the dependence on nuclear masses and write $h(Z)$ and $E_{0}[h(Z)]=-v Z^{2}$ so that the masses are subsumed into the "constant" $v$. Now, when $N=1$, the molecular Hamiltonian becomes

$$
\begin{aligned}
H^{\mathrm{mol}}\left(1, M_{k}, Z_{k}\right)= & -\frac{M_{1}+M_{2}}{2 M_{1} M_{2}} \Delta_{\mathbf{R}}-\frac{1}{2}\left(\frac{1}{m}+\frac{1}{M_{1}+M_{2}}\right) \Delta_{\mathbf{x}} \\
& -\frac{Z_{1}}{\left|\mathbf{x}-\mathbf{R}_{1}\right|}-\frac{Z_{2}}{\left|\mathbf{x}-\mathbf{R}_{2}\right|}+\frac{Z_{1} Z_{2}}{R},
\end{aligned}
$$

and it is easy to see that, whenever $Z_{1} \geqq Z_{2}$,

$$
\inf \left\{\sigma_{\text {ess }}\left(H^{\mathrm{mol}}\right)\right\} \equiv E_{*}\left(H^{\mathrm{mol}}\right)=E_{0}\left[h\left(Z_{1}\right)\right]=-v Z_{1}^{2} .
$$

To construct a partition of unity which localizes to the desired regions, let $\mathbf{x}$ denote the position of the electron and define

$$
F_{0}=g\left(\frac{\varrho}{R}\right), \quad F_{1}=g\left(\frac{R}{\varrho}\right) g\left(\frac{\left|\mathbf{x}-\mathbf{R}_{2}\right|}{\left|\mathbf{x}-\mathbf{R}_{1}\right|}\right), \quad F_{2}=g\left(\frac{R}{\varrho}\right) g\left(\frac{\left|\mathbf{x}-\mathbf{R}_{1}\right|}{\left|\mathbf{x}-\mathbf{R}_{2}\right|}\right) .
$$


with $g$ is as in Appendix B and $\varepsilon=1$. It is easy to check that $F_{0}^{2}+F_{1}^{2}+F_{2}^{2}=1$, and that [compare (B-1) to (B-4)], since $g(t)=0$ if $t<\frac{1}{2}$,

$$
\begin{aligned}
& \operatorname{supp}\left(F_{0}\right) \subset\{(\mathbf{x}, \mathbf{R}): R<2 \varrho\}, \\
& \operatorname{supp}\left(F_{1}\right) \subset\left\{(\mathbf{x}, \mathbf{R}):\left|\mathbf{x}-\mathbf{R}_{1}\right|<2\left|\mathbf{x}-\mathbf{R}_{2}\right| \text { and } \varrho<2 R\right\} \\
& \subset\left\{(\mathbf{x}, \mathbf{R}): R<3\left|\mathbf{x}-R_{2}\right|\right\}, \\
& \operatorname{supp}\left(F_{2}\right) \subset\left\{(\mathbf{x}, \mathbf{R}):\left|\mathbf{x}-\mathbf{R}_{2}\right|<2\left|\mathbf{x}-\mathbf{R}_{1}\right| \text { and } \varrho<2 R\right\} \\
& \subset\left\{(\mathbf{x}, \mathbf{R}): R<3\left|\mathbf{x}-\mathbf{R}_{1}\right|\right\} .
\end{aligned}
$$

The essence of these relations is that $F_{0}$ is non-zero only if the internuclear distance $R$ is small, while $F_{1}\left(F_{2}\right)$ is non-zero only if the electron is closer to the larger (smaller) nucleus. [With $\varepsilon=1$ the overlap of the regions is actually substantial and "closer" means $\left|\mathbf{x}-\mathbf{R}_{1}\right|<2\left|\mathbf{x}-\mathbf{R}_{2}\right|$.] In both cases, the electron is bounded away from the "farther" nucleus by a distance of $\frac{R}{3}$. It follows immediately from (B-7), (9), and (C-1) that the localization error satisfies

$$
\begin{aligned}
L E & \leqq \frac{M_{1}+M_{2}}{M_{1} M_{2}} \frac{b}{\varrho^{2}}+\left(\frac{M_{1}+M_{2}}{M_{1} M_{2}}+\frac{1}{m}+\frac{1}{M_{1}+M_{2}}\right) \frac{b}{R^{2}} g^{2}\left(\frac{R}{\varrho}\right) \\
& \leqq \frac{A_{0}}{M_{2}} \frac{1}{\varrho^{2}} F_{0}^{2}+\frac{A}{M_{2}} \frac{1}{\varrho R}\left(F_{1}^{2}+F_{2}^{2}\right) \equiv L_{0} F_{0}^{2}+L_{1} F_{1}^{2}+L_{2} F_{2}^{2},
\end{aligned}
$$

where this defines $L_{k}$, the property $g^{2}(t)+g^{2}\left(\frac{1}{t}\right)=1$ was exploited to rewrite the inequality in terms of $F_{k}^{2}, A_{0}$ and $A$ are constants, and it was assumed that $M_{1} \geqq M_{2}$.

As a final remark before the proof, note that

$$
\begin{aligned}
& H^{\mathrm{mol}}>h\left(Z_{1}\right)-\frac{Z_{2}}{\left|\mathbf{x}-\mathbf{R}_{2}\right|}+\frac{Z_{1} Z_{2}}{R}=H_{1}+I_{1}, \\
& H^{\mathrm{mol}}>h\left(Z_{2}\right)-\frac{Z_{1}}{\left|\mathbf{x}-\mathbf{R}_{1}\right|}+\frac{Z_{1} Z_{2}}{R}=H_{2}+I_{2},
\end{aligned}
$$

where (for $k=1,2) H_{k}=h\left(Z_{k}\right)$ and the intercluster potentials $I_{k}$ correspond to last two terms. [Compare with (2), (3) and the remark preceding (14).]

As usual, to show that $H^{\text {mol }}$ has no discrete spectrum, it suffices to prove

$$
\begin{aligned}
\left\langle\Psi, H^{\mathrm{mol}} \Psi\right\rangle & =\sum_{k=0}^{2}\left\langle F_{k} \Psi,\left[H^{\mathrm{mol}}-L E\right] F_{k} \Psi\right\rangle \\
& \geqq E_{*}\left(H^{\mathrm{mol}}\right)\|\Psi\|^{2}=-v Z_{1}^{2}\|\Psi\|^{2}, \quad \forall \Psi \in \mathscr{D}\left(H^{\mathrm{mol}}\right),
\end{aligned}
$$

or equivalently, that $\forall \Psi \in \mathscr{D}\left(H^{\mathrm{mol}}\right)$,

$$
\left\langle F_{k} \Psi,\left[H^{\mathrm{mol}}-L_{k}+v Z_{1}^{2}\right] F_{k} \Psi\right\rangle \geqq 0, \quad k=0,1,2 .
$$


The analysis for $F_{0}$ and $F_{1}$ is straightforward. First, the united atom bound implies that

$$
\begin{aligned}
& \left\langle F_{0} \Psi,\left[H^{\mathrm{mol}}-L_{0}+v Z_{1}^{2}\right] F_{0} \Psi\right\rangle \\
& \quad \geqq\left\langle F_{0} \Psi,\left[-v\left\{\left(Z_{1}+Z_{2}\right)^{2}-Z_{1}^{2}\right\}+\frac{Z_{1} Z_{2}}{2 \varrho}-\frac{A_{0}}{M_{2}} \frac{1}{\varrho^{2}}\right] F_{0} \Psi\right\rangle \\
& \geqq\left\langle F_{0} \Psi,\left[-3 v Z_{1} Z_{2}+\frac{Z_{1} Z_{2}}{2 \varrho}-\frac{A_{0}}{M_{2}} \frac{1}{\varrho^{2}}\right] F_{0} \Psi\right\rangle .
\end{aligned}
$$

If $Z_{1} Z_{2}$ is sufficiently large, one can find a constant $d$ so that the last expression will be positive if $\frac{1}{\varrho}=d N^{1 / 3}$. Next, consider the region on which the electron is localized near the larger nucleus. Since $E_{0}\left(F_{1}\right)=-v Z_{1}^{2}$, it follows from (C-10) and (C-6) that

$$
\begin{aligned}
& \left\langle F_{1} \Psi,\left[H^{\mathrm{mol}}-L_{1}+v Z_{1}^{2}\right] F_{1} \Psi\right\rangle \\
& \quad \geqq\left\langle F_{1} \Psi, \frac{1}{R}\left[-3 Z_{2}+Z_{1} Z_{2}-\frac{A}{M_{2} \varrho}\right] F_{1} \Psi\right.
\end{aligned}
$$

which can obviously be made positive if $Z_{1}$ is sufficiently large. $\left(Z_{1}>3\right.$ will suffice if the last term is ignored.)

The analysis for the region on which the electron is localized near the smaller nucleus is more subtle, and contains the essential new idea in the proof. Proceeding as above, one now finds

$$
\begin{aligned}
& \left\langle F_{2} \Psi,\left[H^{\mathrm{mol}}-L_{1}+v Z_{1}^{2}\right] F_{2} \Psi\right\rangle \\
& \quad \geqq\left\langle F_{2} \Psi,\left[v\left(Z_{1}^{2}-Z_{2}^{2}\right)-\frac{3 Z_{1}}{R}+\frac{Z_{1} Z_{2}}{R}-\frac{A}{M_{2}} \frac{1}{\varrho R}\right] F_{2} \Psi\right\rangle .
\end{aligned}
$$

Because of the quadratic term, one would expect this to be positive if $Z_{1}$ is sufficiently large. In order to prove this, first suppose that

Then

$$
\left\langle\Psi, \frac{1}{R} \Psi\right\rangle \leqq v \sqrt{Z_{1}}\|\Psi\|^{2} .
$$

$$
\begin{aligned}
& \left\langle F_{2} \Psi,\left[H^{\mathrm{mol}}-L_{2}+v Z_{1}^{2}\right] F_{2} \Psi\right\rangle \\
& \quad \geqq\left\langle F_{2} \Psi, \frac{1}{R}\left[Z_{1}^{3 / 2}-3 Z_{1}+Z_{1} Z_{2}-\frac{A}{M_{2} \varrho}-\frac{Z_{2}^{2}}{Z_{1}^{1 / 2}}\right] F_{2} \Psi\right\rangle,
\end{aligned}
$$

which will obviously be positive if $Z_{1}$ is sufficiently large. However, we know of no reason to expect $(C-17)$ to be true. Suppose that, instead,

$$
\left\langle\Psi, \frac{1}{R} \Psi\right\rangle \geqq v \sqrt{Z_{1}}\|\Psi\|^{2} .
$$

Then the argument above will not be valid. However, one can use the united atom bound as in (C-14) to conclude that

$$
\begin{aligned}
& \left\langle F_{2} \Psi,\left[H^{\mathrm{mol}}-L_{1}+v Z_{1}^{2}\right] F_{2} \Psi\right\rangle \\
& \geqq\left\langle F_{2} \Psi,\left[-3 v Z_{1} Z_{2}+\frac{Z_{1} Z_{2}}{R}-\frac{A}{M_{2}} \frac{1}{\varrho R}\right] F_{2} \Psi\right\rangle \\
& \geqq\left\langle F_{2} \Psi,\left[-3 v Z_{1} Z_{2}+v Z_{1}^{3 / 2} Z_{2}-\frac{A}{M_{2} \varrho}\right] F_{2} \Psi\right\rangle .
\end{aligned}
$$


Again, this will be positive if $Z_{1}$ is sufficiently large. This completes the proof. If the localization error is ignored, both (C-18) and (C-20) will be positive if $Z_{1}>9$ independent of $Z_{2}$. [ One might try to improve this to $Z_{1}>4$ by noting that 3 could be replaced by $(2+\varepsilon)$ in $(\mathrm{C}-18)$ and by $\left(2+\frac{Z_{2}}{Z_{1}}\right)$ in $(\mathrm{C}-20)$. However, this is even less justifiable as the localization error is $O\left(\varepsilon^{-2}\right)$. In fact, a careful analysis shows that control of the localization error requires $Z_{1}>\frac{c}{Z_{2} M_{2}}=O\left(Z_{2}^{-2}\right)$.

Acknowledgements. Most of this work was done during brief visits to the Mathematisches Forschungsinstitut at Oberwolfach and the ETH in Zürich. It is a pleasure to thank Mr. V. Bach for the benefit of stimulating discussions during these occasions. The author is also grateful to Professor L. Spruch for communicating reference [13] prior to publication, and thereby prompting the author to re-examine the asymmetric one-electron case.

\section{References}

1. Kato, T.: Trans. Am. Math. Soc. 70, 607-630 (1951)

2. Zhislin, G.: Trudy Moskov. Mat. Obsc. 9, 81-128 (1960)

3. Reed, M., Simon, B.: Methods of mathematical physics. IV. Analysis of operators. New York: Academic 1978

4. Thirring, W.: A course in mathematical physics 3. Quantum mechanics of atoms and molecules. Berlin, Heidelberg, New York: Springer 1981

5. Ruskai, M.B.: Commun. Math. Phys. 82, 457-469 (1982); 85, 325-327 (1982)

6. Sigal, I.M.: Mathematical problems in theoretical physics. Seiler, R. (ed.), Lecture Notes in Theoretical Physics, vol. 153, pp. 149-156. Berlin, Heidelberg, New York: Springer 1982; Commun. Math. Phys. 85, 309-324 (1982)

7. Lieb, E.H.: Phys. Rev. Lett. 52, 315-317 (1984); Phys. Rev. A 29, 3018-3028 (1984)

8. Cycon, H.L., Froese, R.G., Kirsch, W., Simon, B.: Schrödinger operators, Sects. 3.7 and 3.8. Berlin, Heidelberg, New York: Springer 1987

9. Ruskai, M.B.: Ann. Inst. H. Poincaré A: Phys. Théorique 52, 397-414 (1990)

10. Ruskai, M.B.: Lett. Math. Phys. 18, 121-132 (1989)

11. Solovej, J.P.: Commun. Math. Phys. 130, 185-204 (1990)

12. Ruskai, M.B., Solovej, J.P.: In preparation

13. Chen, Z., Spruch, L.: Phys. Rev. A 42, 133-138 (1990)

Communicated by B. Simon 